\title{
Energy Management in Hybrid Systems Coupling PV and Electrical Storage
}

\author{
Massimo Brignone, Federico Delfino, Fabio Pampararo, Renato Procopio, Mansueto Rossi and Luca Barillari \\ Dept. of Naval, Electrical, and ICT Engineering \\ University of Genova \\ I-16145, Genova, Italy
}

\begin{abstract}
In this contribution the possible benefits of the integration of a storage system (ST) and a photovoltaic power plant (PV) are investigated by means of a Heuristic Rules System (HRS), used both for the determination of the optimal size of the components and for the power production scheduling. The HRS takes into account the well-known electrical constraints and aims at satisfying the electrical demand following a priority order (PV, ST, main grid). Experimental results in the case of four possible scenarios are presented and discussed.
\end{abstract}

Keywords - energy management; energy efficiency; sustainable microgrids

\section{INTRODUCTION}

The last few years, Photovoltaic (PV) generating units have experienced an extraordinary growth all over the world. In 2012 photovoltaic plants in Europe produced almost 100GW compared to the almost $80 \mathrm{GW}$ in 2011. This increasing trend is expected to continue in the future with a forecasted production of $200 \mathrm{GW}$ for 2020 [1]. Italian PV production has also shown a big increase, rising from $3.5 \mathrm{GW}$ at the end of 2010 to more than $13 \mathrm{GW}$ at the end of 2011 and almost $17 \mathrm{GW}$ at the end of 2012 [2].

This evolution of the electric production mix has changed the operating conditions of the power system, determining some benefits, new problems to be faced and important challenges to be dealt with by Distribution System Operators (DSOs). The need to mitigate the impact of dispersed generation on distribution networks and the level of "market maturity" reached in several countries by technologies such as photovoltaic generation, has accelerated the trend to "grid parity" for renewable generation (i.e. lower feed-in tariffs, higher difference between purchasing and selling prices) [3],[4],[5]. This, in turn, has moved the application focus from plants primarily intended for power production to be sold to the network to systems devoted to self-consumption. However, since PV units cannot be dispatched but depend on the availability of the primary energy source and energy must provided to the load whenever it requires it, the selfconsumption mode has increased the need for reliable storage systems and effective integration schemes between PV units and storage devices. Thanks to this new operation conditions, storage has got new potential applications into:

- renewables integration: maximized benefits in the market, improved fit to predictions, repowering over existing infrastructures, maximization of capacity factor

- ancillary services for the grid: reactive and active power control for voltage and frequency regulation

- load/generation shifting and peak shaving

- quality of supply and emergency power in case of a long duration contingency.

As a result, manufacturers are proposing now a number of solutions, targeted also to residential applications, typically composed by a power converter plus a storage system, to be used in conjunction with renewable generation and eventually gensets [6],[7].

In this framework, when storage systems are integrated with renewable plants and connected to the distribution grid, there is a need to develop advanced control systems to meet the requirements demanded by the grid and the market. Control algorithms must account for the existing business model, respond to the functional specifications developed in the design stage and take into account the restrictions coming from technologies not fully developed and known. That opens a wide area of innovations related to both control and SCADA systems for storage and hybrid systems management.

In this paper the economic benefits coming from the integration of a storage system in a renewable power plant are investigated by means of a simple Heuristic Rules System (HRS), based on the availability of renewable generation and on the power demand at each time.

The paper is organized as follows: in Section II the HRS logics is described from a theoretical point of view, Section III is devoted to present numerical results and finally some conclusions are drawn in Section IV. 


\section{LOGIC DSCRIPTION}

In this section the HRS strategy is presented. In the following, a set of $N_{t}$ time intervals will be considered to define the time horizon: $\Delta t$ is the length of each time interval $t$, which goes from time $t$ to $t+\Delta t$.

The rule we are considering can be graphically visualized in Fig. 1.
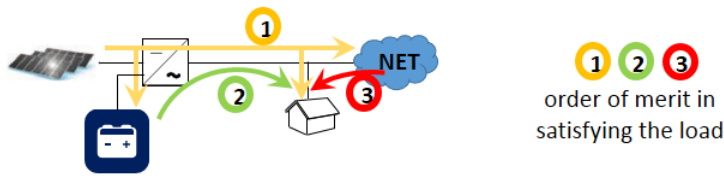

Fig. 1. HRS logic scheme

More precisely the procedure can be described as [6]:

- if renewable generation power is greater than demand: charge the battery if not completely charged, otherwise sell the exceeding energy to the main grid;

- if renewable generation power is less than demand and the state of charge of the battery is greater than minimum: discharge the battery and if it is not sufficient absorb power from the main grid;

- if renewable generation power is less than demand, and minimum charge of the battery is reached: absorb power from the main grid.

In order to apply the HRS, a model of the photovoltaic unit as well as of the storage system has to be developed.

The PV unit is basically described at time $t$ by one variable: the active power, denoted by $\mathrm{P}_{\mathrm{PV}, t}$, which is function of the solar irradiance and the temperature [8], and is always less than the peak power $\mathrm{P}_{\mathrm{PV}, \max }$.

The storage system (ST) is represented by the injected active power, $P_{S T, t}$, and the storage energy content, $W_{S T, t}$ [9]. The injected active power depends on $W_{S T, t}$ through a piecewise linear relationship involving two performance

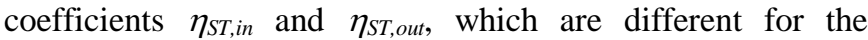
charging and discharging of the battery, as follows:

$$
P_{S T, t}=\left\{\begin{array}{cc}
\frac{1}{\eta_{S T, \text { in }}} \frac{W_{S T, t-1}-W_{S T, t}}{\Delta t} & W_{S T, t-1}<W_{S T, t} \\
\eta_{S T, \text { out }} \frac{W_{S T, t-1}-W_{S T, t}}{\Delta t} & W_{S T, t-1} \geq W_{S T, t}
\end{array}\right.
$$

Of course, all the involved quantities are subjected to suitable bounds:

$$
W_{S T, \text { min }} \leq W_{S T, t} \leq W_{S T, \text { max }}
$$

where $W_{S T \text {,min }}$ and $W_{S T \text {, max }}$ represent the minimum and the maximum value for the stored energy, respectively; similarly

$$
P_{S T, c h} \leq P_{S T, t} \leq P_{S T, d s c h}
$$

where $P_{S T, c h}$ and $P_{S T, d s c h}$ represent the maximum power during the charging phase (negative number, according to the aforementioned conventions) and the maximum power during the discharging phase (positive number).

Once known the Electric Loads at time $t$ (described as negative active power injections $\left.P_{E L, t}\right)$ and the photovoltaic power production at time $t$ (described as a positive power $\left.P_{P V, t}\right)$, it is possible to compute the overall energy cost, as follows:

$$
C_{t o t}=\sum_{t=1}^{N_{t}} C_{N E T, t}+C_{s e l l, N_{t}}\left(W_{S T, N_{t}}-W_{S T, \min }\right)
$$

Here the last term in the cost function would formalize the virtual gain coming from the possible amount of energy stored at the end of the analyzed period. The cost/gain coming from the energy buying/selling from the Distribution System Operator (DSO) is expressed as

$$
C_{N E T, t}= \begin{cases}C_{\text {sell }, t} P_{N E T, t} \Delta t & P_{N E T, t}<0 \\ C_{\text {buy }, t} P_{N E T, t} \Delta t & P_{N E T, t} \geq 0\end{cases}
$$

where $C_{\text {sell,t }}$ and $C_{\text {buy,t }}$ represent respectively the cost, depending on the time slot, of the sold and purchased energy, while $P_{N E T, t}$ is the power injected into the system by the external network at time $t$, and is determined by the electric balance equation, assuming a single bus bar model for the network:

$$
P_{N E T, t}+P_{S T, t}+P_{P V, t}+P_{E L, t}=0
$$

The cost in (4) together with the described constraints can be used as the starting point to create an optimization procedure able to provide the better size of the photovoltaic power plant and the storage system, once the forecasted electrical loads, the solar irradiance and the temperature curves are at disposal, (or equivalently, using [8], the forecasted the power production per unitary peak photovoltaic power plant). In fact, if introduced $C_{S T}$ (the cost per installed $\mathrm{kWh}$ per hour) and CPV (the cost per installed peak $\mathrm{kW}$ per hour), the optimization procedure aims at minimizing

$$
C=C_{t o t}+\left(C_{P V} P_{P V, \text { max }}+C_{S T} W_{S T, \text { max }}\right) N_{t} \Delta t
$$

in the two free variables $P_{P V, \max }$ and $W_{S T, \max }$.

\section{NUMERICAL RESULTS}

In this section some numerical results are shown on four possible applications: a residential, a small industry, a medium industry and a large one. To compute the optimal size of 
storage system and photovoltaic power plant, the procedure described in Section II has to be applied to a large set of data: to this aim we considered one year of real photovoltaic power production and electrical loads profile in order to define four typical days (one for each season) mediating the dataset. In Fig. 2 the power production per unitary peak photovoltaic power plant are displayed (that curves are obtained by measurements of the Savona Campus Smart Poligeneration Microgrid [11]). The electrical load curves, for each case under analysis, can be read in Figs. 3-4-5 (dotted line).

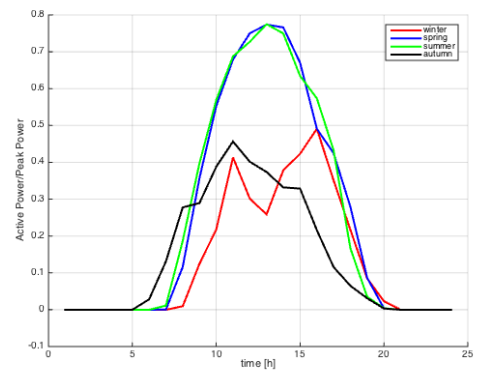

Fig. 2. PV generation per unitary $\mathrm{kW}$ installed for a day per each season

The used profiles have been replicated in order to evaluate the cost/gain in the period of a whole year.

For the optimization procedure the cost of the storage system is quantified in $500 € / \mathrm{kWh}$ to be subdivided onto a mean life of 4000 days [12] (i.e. $C_{S T}=0.0052 € /(\mathrm{kWh} \cdot \mathrm{h})$ ) and the cost of the photovoltaic power plant in $3000 € / \mathrm{kW}$ to be subdivided onto a mean life of 20 years [13] (i.e. $C_{P V}=0.0171$ $€ /(\mathrm{kW} \cdot \mathrm{h}))$. Moreover, the storage system has the following technical characteristics: mean performance coefficients $\eta_{S T, \text { in }}=1.0$ and $\eta_{S T, \text { out }}=0.8$, the maximum power allowed in both

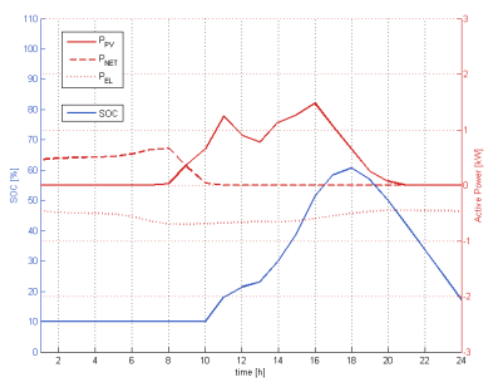

(a)

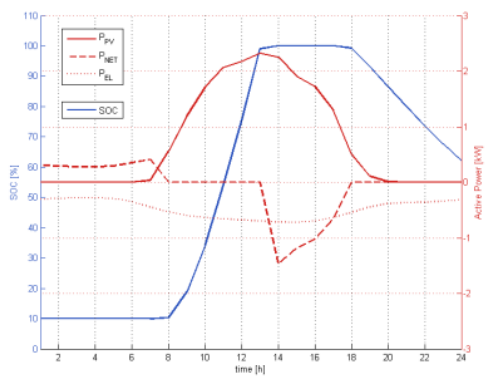

(c) the charging and discharging phases are $P_{S T, c h}=-(0.3$ $\left.\mathrm{h}^{-1}\right) W_{S T, \max }$ and $P_{S T, d s c h}=\left(0.3 \mathrm{~h}^{-1}\right) W_{S T, \max }$, the Depth of Discharge $(\mathrm{DoD})$ is $[10 \%, 100 \%]$, i.e. $W_{S T, \min }=0.1 W_{S T, \max }$. According to the Italian DSO we assume $C_{\text {sell, },}=0.08 € / \mathrm{kWh}$ and $C_{b u y, t}=0.256 € / \mathrm{kWh}$ if the time $t$ is between 8:00 AM and 8:00PM, otherwise $C_{b u y, t}=0.198 € / \mathrm{kWh}$. Under these assumptions, the results of the optimization procedure are collected in Table I: for each case of interest the size of the storage system and the photovoltaic unit, the annual cost and the saving percentage with respect the case where nothing is installed are presented. In Figs. 3-4-5 also the State of Charge (SOC) of the optimal storage system, the power profile of the optimal power plant and the power profile exchanged with the main grid obtained using the HRS are displayed, for all the four cases of interest and for each typical day selected.

It can be easily deduced by Table I that, for each considered case, the presence of a photovoltaic power plant and a storage system permits to save from $35 \%$ up to $47 \%$ of the annual expense.

TABLE I. VALUES OF THE OPTIMAL SIZE OF PHOTOVOLTAIC POWER PLANT AND SOTRAGE SYSTEM, ANNUAL COSTS AND SAVING PERCENTAGE FOR THE FOUR CASES OF INTEREST.

\begin{tabular}{|c|c|c|c|c|}
\hline & PV size & ST size & $\begin{array}{c}\text { Annual } \\
\text { Cost }\end{array}$ & \% saving \\
\hline Residential & $3 \mathrm{~kW}$ & $1 \mathrm{kWh}$ & $2174 €$ & $37.7 \%$ \\
\hline $\begin{array}{c}\text { Small } \\
\text { industry }\end{array}$ & $60 \mathrm{~kW}$ & $20 \mathrm{kWh}$ & $44076 €$ & $41.4 \%$ \\
\hline $\begin{array}{c}\text { Medium } \\
\text { industry }\end{array}$ & $180 \mathrm{~kW}$ & $80 \mathrm{kWh}$ & $117778 €$ & $46.9 \%$ \\
\hline $\begin{array}{c}\text { Large } \\
\text { industry }\end{array}$ & $1200 \mathrm{~kW}$ & $300 \mathrm{kWh}$ & $903375 €$ & $35.1 \%$ \\
\hline
\end{tabular}

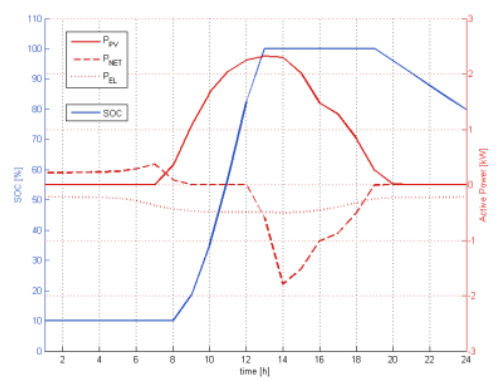

(b)

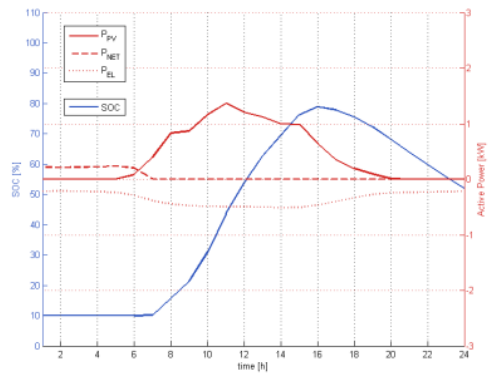

(d)

Fig. 3. HRS profiles for residential application. Electrical loads, photovoltaic power production, network power exchange and SOC for a typical day of winter (a), spring (b), summer (c) and autumn (d) 


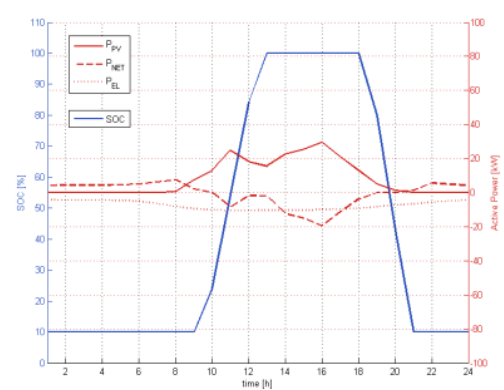

(a)

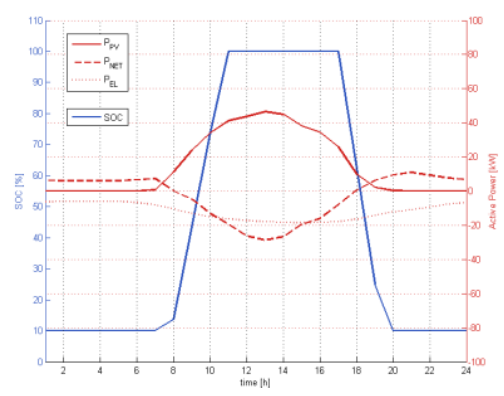

(c)

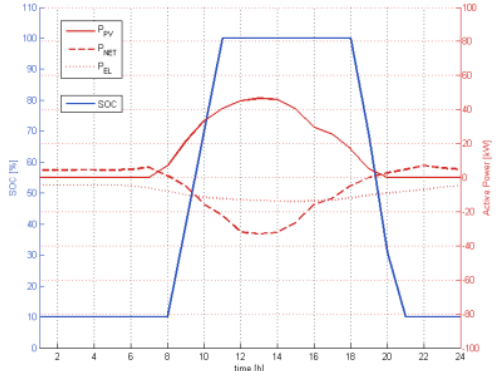

(b)

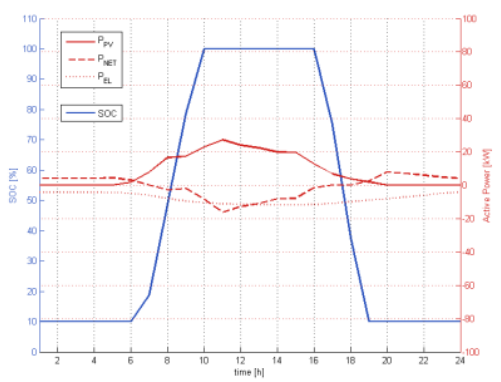

(d)

Fig. 4. HRS profiles for small-industry application. Electrical loads, photovoltaic power production, network power exchange and SOC for a typical day of winter (a), spring (b), summer (c) and autumn (d)

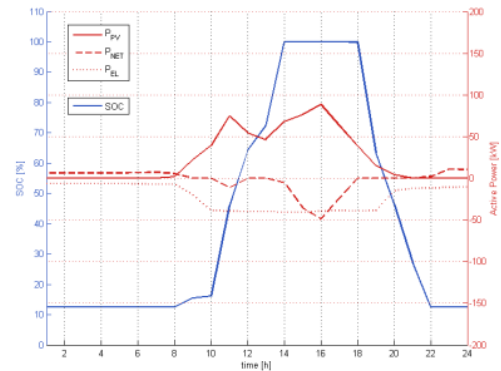

(a)

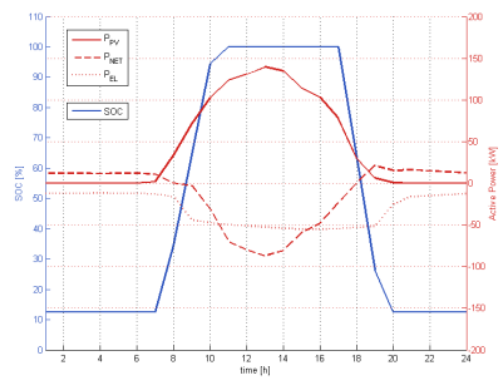

(c)

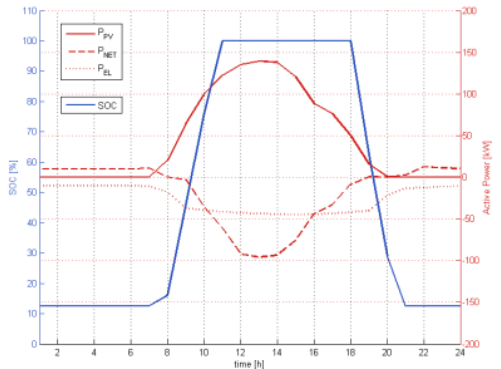

(b)

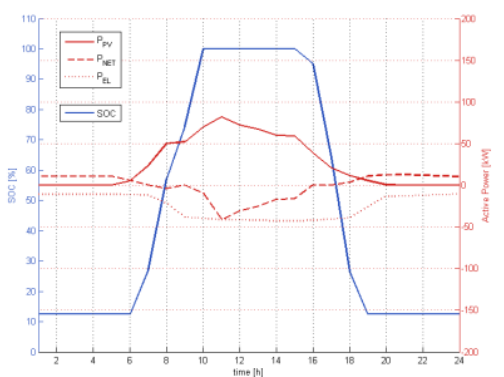

(d)

Fig. 5. HRS profiles for medium-industry application. Electrical loads, photovoltaic power production, network power exchange and SOC for a typical day of winter (a), spring (b), summer (c) and autumn (d) 


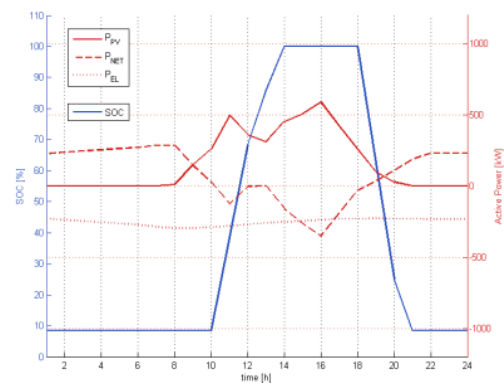

(a)

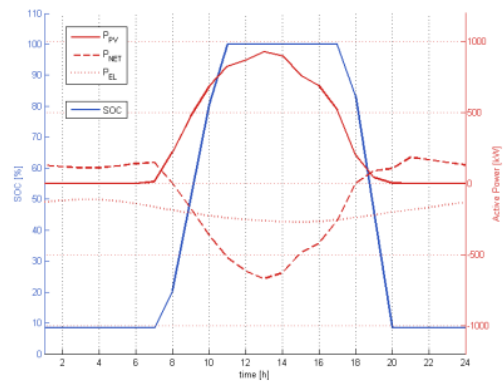

(c)

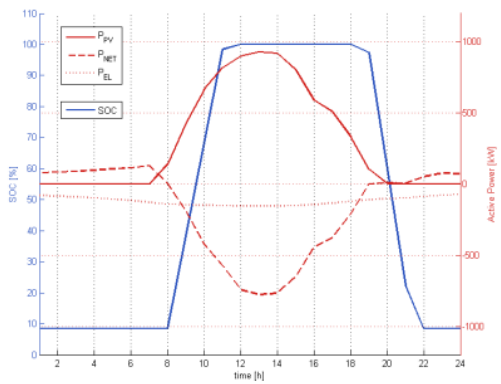

(b)

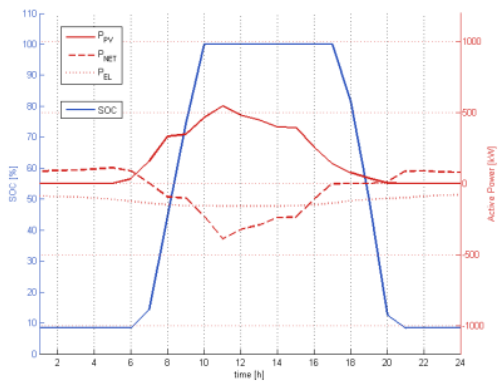

(d)

Fig. 6. HRS profiles for large- industry application. Electrical loads, photovoltaic power production, network power exchange and SOC for a typical day of winter (a), spring (b), summer (c) and autumn (d)

\section{CONCLUSIONS}

In the paper, a simple logic (HRS) for the management of a grid has been proposed, which integrates a storage system and a photovoltaic unit. More precisely, HRS satisfies the electrical demand following a priority order and encodes the constrains due to the electrical active power balance and physical model relations. A cost function that involves the energy cost due to the buying/selling to the external network and a virtual gain due to the stored energy, as well as the setup of the system is defined. This permitted to build-up an optimization procedure that has quantified the benefit of the introduction of photovoltaic power plant and a storage system in four possible scenarios ranging from residential to large industry plants.

\section{REFERENCES}

[1] "Solar Photovoltaic on the road to Large-Scale Grid Integration," EPIA, Tech. Rep., Sep. 2012. [online] Available: http:// www.epia.org.

[2] "Activity of the year 2011," GSE, Tech. Rep., 2011. [online] Available:http://www.gse.it/it/Dati\%20e\%20Bilanci/pages/default.aspx

[3] M. Castillo-Cagigal, E. Caamaño-Martín, D. Masa-Bote, A. Gutierrez, F. Monasterio-Huelin, and J. Jimenez-Leube, "PV self-consumption optimization with storage and Active DSM for the residential sector," Solar Energy, vol. 85, no. 9, pp. 2338-38, 2011.

[4] Self consumption of PV electricity, European Photovoltaic Industry Association (EPA) position paper, 2013.
[5] N. Femia, D. Toledo, and W. Zamboni "Storage unit and load management in photovoltaic inverters for residential application," in Industrial Electronics Society, IECON 2013 - 39th Annual Conference of the IEEE, pp. 6800-6805.

[6] Sirio Power Supply, AROS solar technology, [online] Available: http://www.aros-solar.com/en/sirio-power-supply-sps

[7] StoraXe Energy Storage Solutions, ads-tec GmbH, [online] Available: http://www.ads-tec.de/en/energy-storage/home-small-business.html.

[8] U. Boke, "A Simple model of photovoltaic module electric characteristics" European Conference on Power Electronics and Applications (Aalborg, Denmark), 2007, pp. 1-8.

[9] S. Bracco, M. Brignone, F. Delfino, and R. Procopio, "An Energy Management System for the Savona Campus Smart Polygeneration Microgrid," IEEE Systems Journal, to appear, 2015.

[10] IBM. IBM ILOG CPLEX Optimization Studio. Software, 2012. [online] Available: http://www-01.ibm.com/software/integration/optim ization/cplex-optimization-studio.

[11] A. Bonfiglio, L. Barillari, M. Brignone, F. Delfino, F. Pampararo, R. Procopio, M. Rossi, S. Bracco, and M. Robba, "An optimization algorithm for the operation planning of the University of Genoa smart polygeneration microgrid," Bulk Power System Dynamics and Control IX Optimization, Security and Control of the Emerging Power Grid (IREP2013), pp. 1-8.

[12] FIAMM, SoNick battery for Energy Storage Systems [online], http://www.fiamm.com/media/223003/20150209_st523_datasheet.pdf.

[13] D. Feldman, G. Barbose, R. Margolis, T. James, S. Weaver, N. Darghouth, R. Fu1, C. Davidson, S. Booth, and R. Wiser, "Photovoltaic System Pricing Trends," [online] http://www.nrel.gov/docs/fy14osti/ 62558.pdf. 